\title{
Trois espèces d'Oxyures parasites de Poissons en Afrique
}

\section{par Annie-J. PETTER, Georges VASSILIADES et Pierre-Maurice TRONCY}

Laboratoire de Zoologie (Vers), associé au C.N.R.S. ( $\mathrm{P}^{\mathrm{r}}$ A.-G. ChaBaud), Muséum national d'Histoire naturelle, 57, rue Cuvier, 75005 Paris

Laboratoire national de Recherches vétérinaires, Dakar, Sénégal, et Laboratoire de Farcha, B.P. 433, Fort-Lamy, République du Tchad

\section{Résumé}

Trois espèces d'Oxyures ont été trouvées dans les tubes digestifs de Poissons d'eau douce en Afrique:

- Cithariniella citharini Khalil 1964 trouvée chez Citharinus citharus (Geoffroy) et Distichodus brevipinnis Günther au Sénégal.

- Cithariniella khalili n. sp., trouvée chez Synodontis sorex Günther au Sénégal et Synodontis gambiensis latifrons Blache au Tchad. Elle se distingue de l'espèce précédente principalement par sa queue plus courte et sa vulve plus antérieure.

- Synodontisia thelastomoides n.g., n.sp., trouvée chez Synodontis sorex au Sénégal et Synodontis ocellifer Boulenger au Tchad. Le genre est caractérisé principalement par 3 paires de petites papilles concentrées autour du cloaque et une papille double impaire sur la queue.

Les deux genres Cithariniella et Synodontisia présentent beaucoup de ressemblances avec certains Thelastomatidae et nous supposons qu'ils représentent une lignée issue par capture de ceux-ci et ayant peu évolué depuis. Nous considérons que l'ensemble des Pharyngodoninae groupe en réalité des genres polyphylétiques issus à plusieurs reprises des Oxyures d'Insectes.

\section{Summary}

Three species of Oxyuridae were found in the gut of freshwater fishes in Africa:

- Cithariniella citharini Khalil 1964, from Citharinus citha. rus (Geoffroy) and Distichodus brevipinnis Günther. 
- Cithariniella khalili n. sp., from Synodontis sorex Günther and Synodontis gambiensis latifrons Blache. It is distinguished from Cithariniella citharini by its shorter tail and more anterior vulva.

- Synodontisia thelastomoides n. g., n. sp., from Synodontis sorex Günther and Synodontis ocellifer Boulenger. The genus is characterized by three pairs of small papillae gathered around the cloaca and one double papilla on the tail.

The two genera Cithariniella and Synodontisia show similarities with some Thelastomatidae and we suppose they are issued from them by \&capture » and have evolved but little since. Pharyngodoninae are considered as a group of polyphyletic genera issued at different times from Insect Oxyurids.

Le matériel étudié provient des tubes digestifs de Poissons pêchés respectivement dans le lac de Guiers (Sénégal) et dans le fleuve Chari à Fort-Lamy (Tchad).

Nous avons trouvé trois espèces différentes d'Oxyures, dont deux cohabitent chez le même hôte.

\section{I. - Cithariniella citbarini Khalil 1964 (fig. 1)}

Cette espèce a été trouvée au Sénégal (lac de Guiers), chez Citharinus citharus (Geoffroy) et Distichodus brevipinnis Günther.

MATÉRIEL ÉTUdIÉ.

Chez Distichodus brevipinnis 292 BA : plusieurs femelles adultes.

Chez Citharinus citharus 293 BA : quelques femelles juvéniles sans œufs, et deux larves mâles du quatrième stade préparant la dernière mue.

Khalil décrit, en 1964, au Soudan, chez Citharinus citharus, une espèce, Cithariniella citharini, dont les femelles, quoique légèrement plus grandes que les nôtres, présentent les mêmes proportions relatives et les mêmes caractères morphologiques; le $\mathrm{D}^{r}$ Khalil a eu l'obligeance de nous envoyer quelques-uns de ses spécimens, et nous avons pu vérifier l'identité des deux lots.

FIG. 1. - Cithariniella citharini Khalil, 1964. - A. - femelle, vue latérale. B. - femelle, vue apicale superficielle. C. - femelle, coupe optique au niveau des pièces chitinoïdes striées de la cavité buccale. D. - femelle, extrémité antérieure, vue latérale. E. - portion distale de l'ovéjecteur. F. - œuf. G. - ébauche génitale chez une larve femelle. H. - région anale d'une larve mâle montrant les papilles de l'adulte sous la cuticule larvaire. I. - épaississement cuticulaire de la queue du mâle, vue ventrale (dessin effectué sur un spécimen confié par Khalil). (A : échelle 500 u. B B, C, H: échelle $20 \mu ; \mathrm{E}, \mathrm{G}, \mathrm{I}$ : échelle $100 \mu$; D, F : échelle $50 \mu$ ). 
OXYURES PARASITES DE POISSONS AFRICAINS
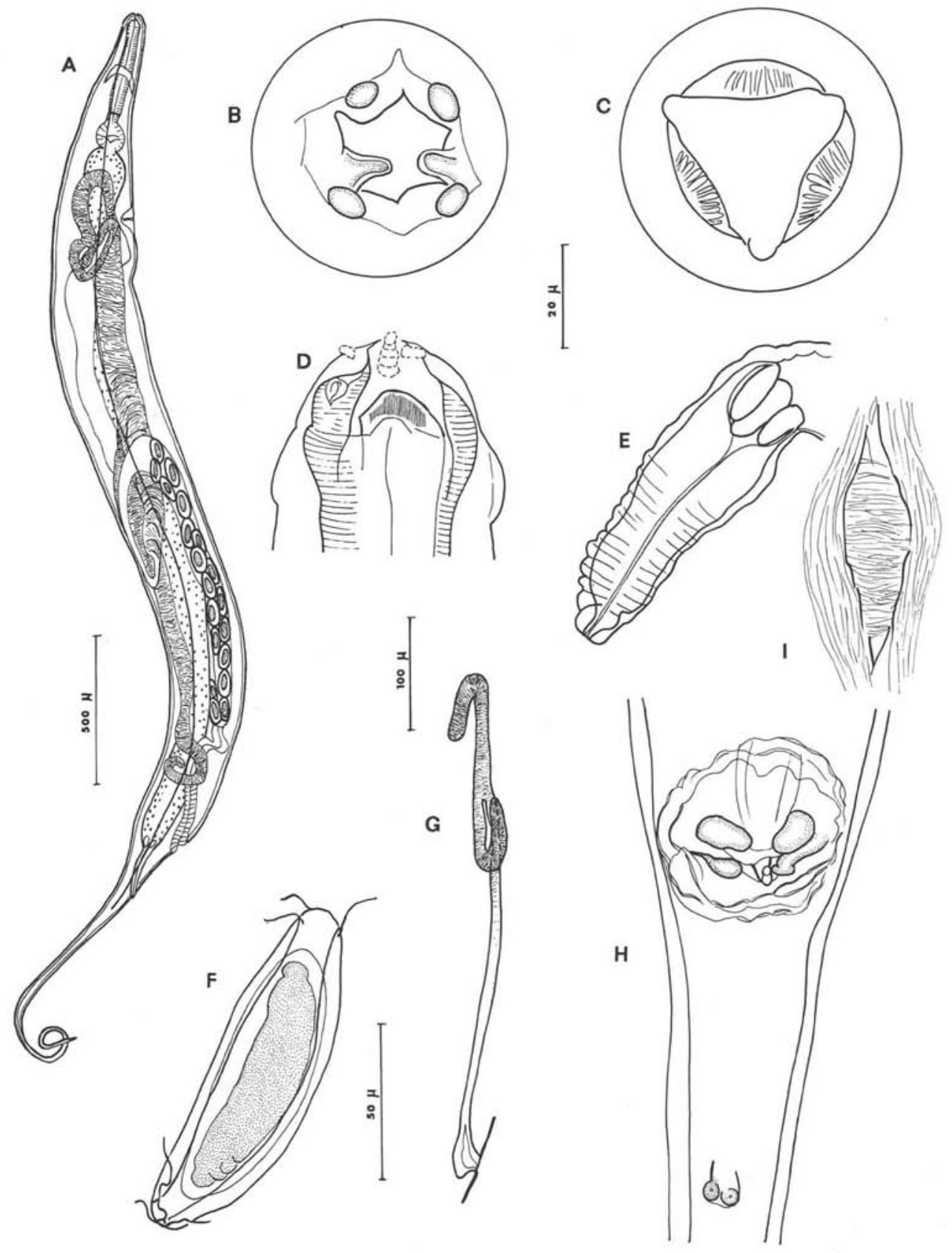
L'espèce étant déjà décrite, nous donnons simplement quelques compléments morphologiques pour faciliter la comparaison avec les espèces suivantes.

L'extrémité antérieure, conique, est légèrement enflée par rapport au reste du corps, formant une «tête » globuleuse, elle-même divisée en deux transversalement par un léger sillon ; la bouche présente six lèvres, quatre larges lèvres submédianes et deux étroites lèvres latérales parcourues sur toute leur longueur par les amphides allongées ; il existe quatre grosses papilles submédianes ovalaires doubles.

Nous avons observé d'étroites ailes latérales (larges de $10 \mu$ ) qui débutent environ au niveau du quart antérieur de l'œsophage, et s'étendent jusqu'un peu en dessous de l'anus chez les femelles.

L'une des larves du quatrième stade, longue de $1.650 \mu$, montre sous la cuticule larvaire une région cloacale, portant deux grosses paires de papilles allongées encadrant l'ouverture cloacale, située à $450 \mu$ de l'extrémité ; à $70 \mu$ en dessous du cloaque, on observe ventralement sous la cuticule larvaire une paire de papilles arrondies contiguës.

Principales dimensions d'une femelle longue de $4 \mathrm{~mm}$.

Largeur maximum : $300 \mu$; cavité buccale: $30 \mu$ de profondeur; œsophage: $450 \mu$; anneau nerveux et pore excréteur situés respectivement à $180 \mu$ et $675 \mu$ de l'extrémité antérieure ; queue : $1100 \mu$; vulve située à $180 \mu$ en avant de l'anus ; œufs : $110 \mu / 35 \mu$.

\section{II. - Cithariniella kbalili n. sp. (fig. 2)}

Cette espèce a été trouvée associée à l'espèce suivante, au Sénégal (lac de Guiers) chez Synodontis sorex Günther, et au Tchad (fleuve Chari, Fort-Lamy) chez Synodontis gambiensis latifrons Blache.

MATÉRIEL ÉTUdí́.

Chez Synodontis gambiensis 295 BA (matériel type) : un mâle et quelques femelles.

Chez Synodontis sorex 291 BA : un mâle et quelques femelles.

\section{DESCRIPTION.}

Oxyures de petite taille, à corps cylindrique, à queue effilée dans les deux sexes. La striation cuticulaire est fine et peu marquée (interstries de $2 \mu$ ), et on n'observe pas d'annélation superficielle visible; il existe d'étroites ailes latérales (larges d'environ $10 \mu$.$) .$

FIg. 2. - Cithariniella khalili n. sp. A. - femelle, vue latérale. B. - femelle, vue apicale superficielle. C. - femelle, coupe optique au niveau des pièces chitinoïdes striées de la cavité buccale. D. femellc, extrémité antérieure, vue latérale. E. - mâle, vue latérale. F. - mâle, vue apicale superficielle. G. - mâle, régioñ cloacale, vue latérale. H. - mâle, extrémité caudale, vue ventrale. I. portion distale de l'ovéjecteur. J. - œuf. (A, E: échelle $500 \mu$; B, C, D, F : échelle $20 \mu$; G, H, J : échelle $50 \mu ; \mathrm{I}$ : échelle $\left.100_{\mu}\right)$ 


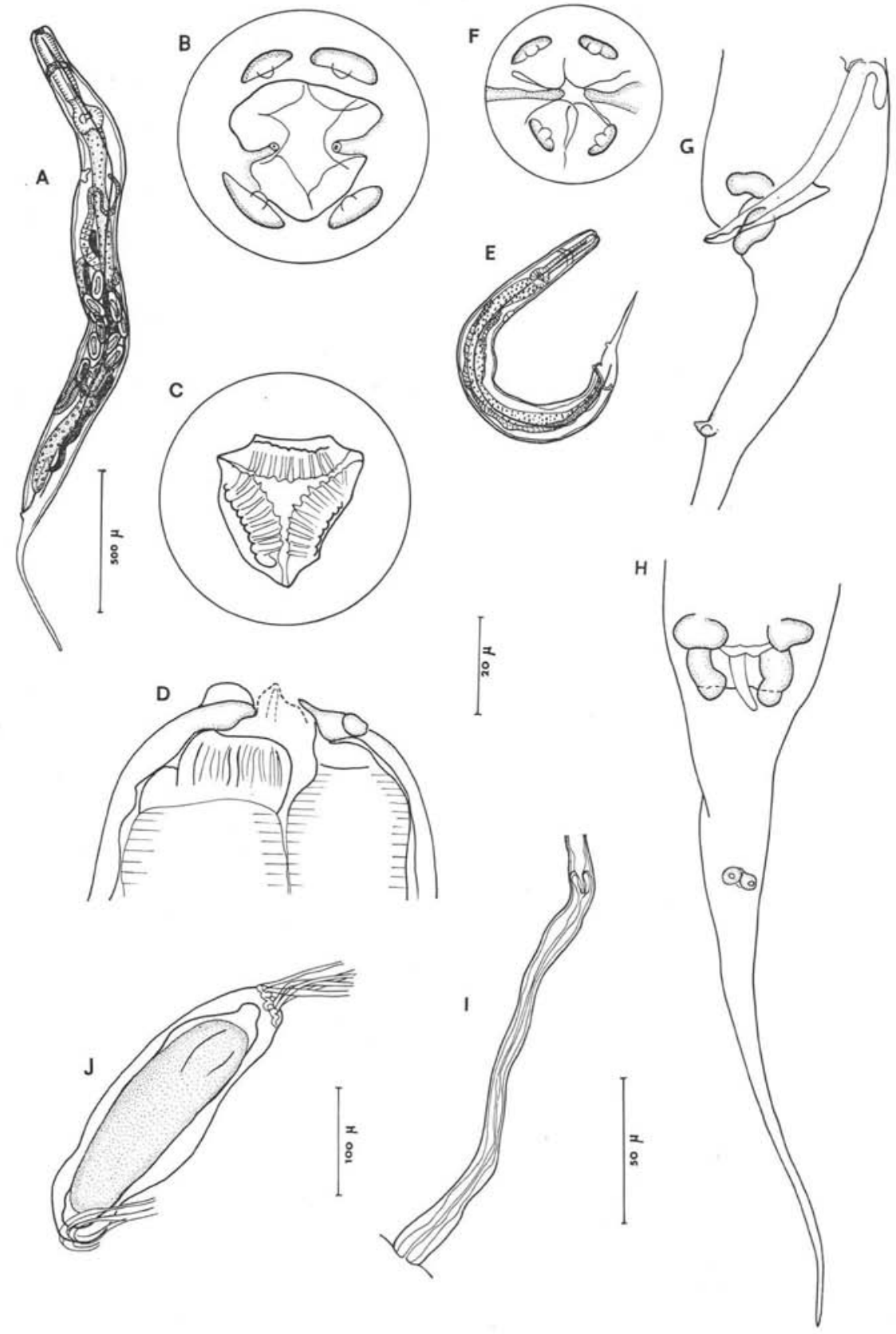


La bouche présente six lèvres, quatre submédianes et deux latérales qui sont plus étroites et parcourues sur toute leur longueur par les amphides allongées; les quatre grosses papilles submédianes portent un petit mamelon à leur sommet. La bouche s'ouvre dans une cavité buccale au fond de laquelle se dressent trois pièces chitinoïdes striées.

Esophage cylindrique, avec isthme court et bulbe sphérique valvulé.

\section{Femelles.}

La vulve est située environ aux deux tiers de la longueur du corps ; l'ovéjecteur, dirigé vers l'extrémité antérieure, comprend une première portion à paroi épaisse (longue de $400 \mu$ environ), terminée par un sphincter, et une deuxième portion à paroi mince emplie d'œufs, qui se divise à son extrémité en deux utérus opposés.

Les œufs sont allongés ; à chacun de leurs pôles s'insèrent cinq filaments relativement épais ; ces filaments sont très longs, et l'ensemble des filaments de tous les œufs se rassemble pour former des écheveaux qui parcourent les utérus et la deuxième partie de l'ovéjecteur.

La queue est longue et effilée.

Principales dimensions d'une femelle longue de $2.300 \mu$.

Largeur maximum: $160 \mu$; cavité buccale : $20 \mu$ de profondeur ; œsophage : $400 \mu$; anneau nerveux et pore excréteur situés respectivement à $150 \mu$ et $540 \mu$ de l'extrémité antérieure; vulve située à $860 \mu$ de l'extrémité postérieure ; queue : $500 \mu$; ailes latérales débutant à $40 \mu$ de l'extrémité antérieure et se terminant au niveau du rectum ; œufs : $110 \mu / 35 \mu$.

\section{Mâles.}

La queue des mâles porte, environ au quart antérieur de sa longueur, une paire de papilles contiguës ; au-delà de ces papilles, la queue devient très effilée ; il existe deux grosses paires de papilles cloacales, une paire large immédiatement au-dessus du cloaque, et une paire allongée immédiatement en dessous; le spicule est long et aciculaire, avec un capitulum recourbé dorsalement; il existe un petit gubernaculum accolé dorsalement au spicule, dans sa région distale.

Principales dimensions d'un mâle long de $1.550 \mu$.

Largeur maximum : $100 \mu$; œsophage : $275 \mu$; anneau nerveux et pore excréteur situés à $110 \mu$ et $440 \mu$ de l'extrémité antérieure; queue: $260 \mu$ avec papilles caudales situées à $200 \mu$ de l'extrémité ; spicule : $85 \mu$; gubernaculum : $25 \mu$; ailes latérales débutant à $40 \mu$ de l'extrémité antérieure et se terminant au niveau du cloaque.

\section{Discussion.}

Notre espèce présente avec Cithariniella citharini de grandes affinités: même structure apicale, mêmes pièces chitinoïdes striées dans la cavité buccale, même aspect des papilles caudales chez le mâle avec deux paires de grosses papilles autour du 
cloaque et une plus postérieure saillante, spicule recourbé en crosse à son extrémité proximale, vulve située postérieurement et œufs munis de filaments ; nous plaçons donc notre espèce dans le genre Cithariniella; elle présente cependant, avec l'espèce de Khalil, un certain nombre de différences: plus petite taille, queue plus courte dans les deux sexes, vulve moins proche de l'anus, absence d'épaississement cuticulaire sur la face ventrale du mâle. L'espèce est donc nouvelle et nous la nommons Cithariniella khalili n. sp.

\section{III. - Synodontisia thelastomoides n. g., n. sp. (fig. 3)}

Cette espèce a été trouvée à la fois au Sénégal (lac de Guiers), chez Synodontis sorex Günther, où elle était associée à l'espèce précédente, et au Tchad (fleuve Chari, Fort-Lamy) chez Synodontis ocellifer Boulenger.

MATÉRIEL ÉtUdí́.

Chez Synodontis sorex 291 BA (matériel type): deux femelles mûres et un mâle en mauvais état.

Chez Synodontis ocellifer 294 BA : quelques femelles dont plusieurs juvéniles et un mâle dont la région cloacale est abîmée.

\section{DESCRIPTION.}

Oxyures de petite taille à corps en fuseau, à queue très longue et effilée dans les deux sexes. La cuticule présente une striation transversale très marquée, à stries relativement espacées (interstries de $20 \mu$ à $30 \mu$ ) formant une annélation superficielle nette ; cette striation débute à $40 \mu$ de l'apex ; il existe des ailes latérales étroites (une vingtaine de $\mu$ ) débutant un peu en avant de l'anneau nerveux et se terminant à peu près au niveau de l'anus chez les femelles et à peu près au milieu de la queue chez les mâles.

La bouche présente six lèvres: quatre lèvres submédianes larges et deux lèvres latérales plus étroites. On observe quatre grosses papilles submédianes ovalaires et deux amphides allongées qui parcourent toute la longueur des lèvres latérales. La bouche s'ouvre dans une petite cavité buccale limitée par les parois amincies de l'œsophage ; l'œsophage présente un corpus cylindrique, un isthme court et un bulbe sphérique valvulé.

\section{Femelles.}

Le diamètre des femelles varie beaucoup suivant l'âge, les femelles mûres et pleines d'œufs ayant le corps très élargi. 

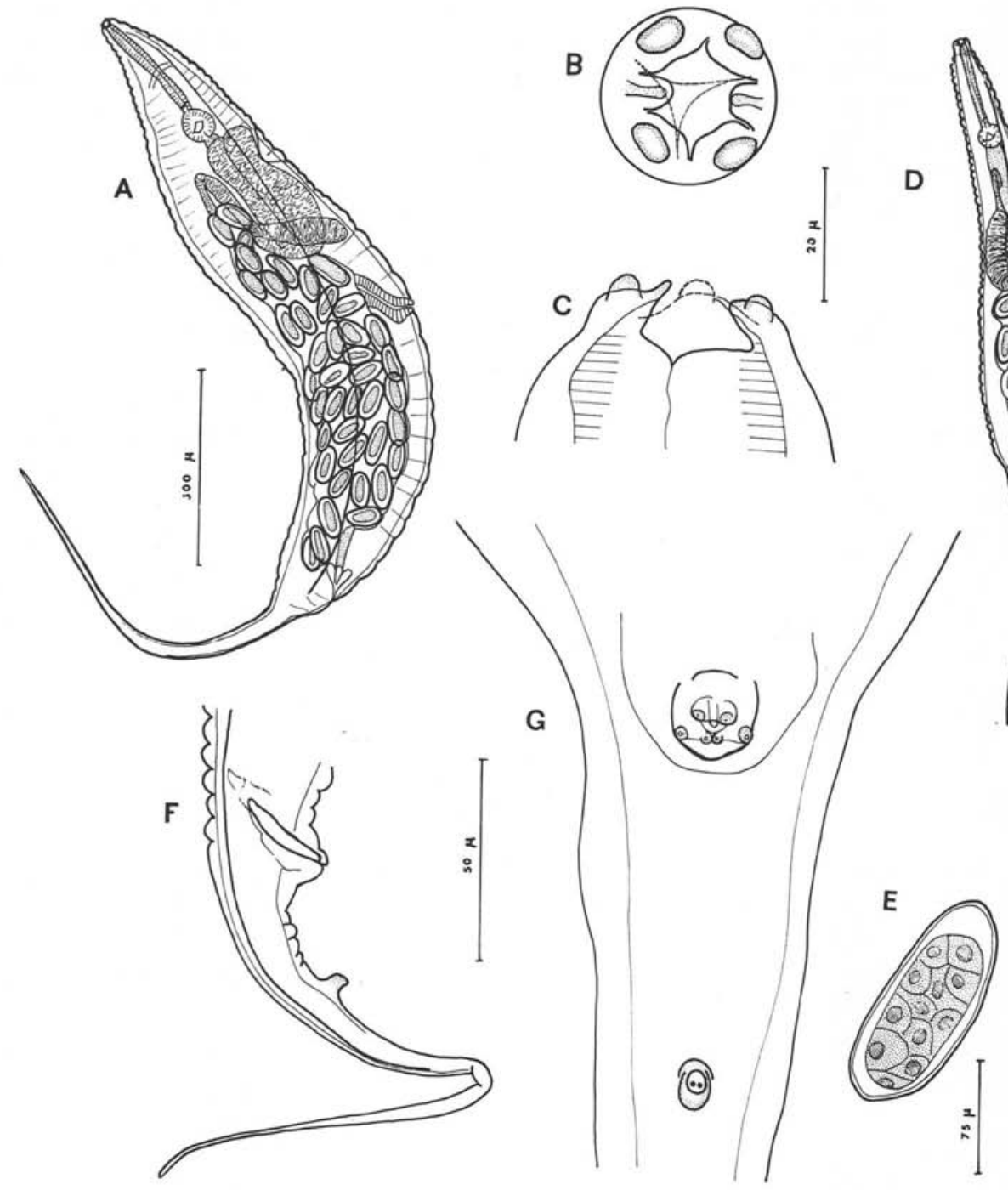

FIG. 3. - Synodontisia thelastomoides n. g., n. sp. A. - femelle, vue latérale. B. - femelle, vue apicale superficielle. C. - femelle, extrémité antérieure, vue submédiane. D. - femelle juvénile, vue latérale. E. - œuf. F. - mâle, extrémité postérieure, vue latérale. G. - mâle, région cloacale, vue ventrale. (A, D : échclle $50 \mu_{\mu} ; \mathrm{B}, \mathrm{C}$ : échelle $20_{\mu} ; \mathrm{E}, \mathrm{F}$ : échelle $75 \mu$; G : échelle $50 \mu$ )

La vulve est située un peu en avant du milieu du corps; l'ovéjecteur est d'abord dirigé vers l'avant, puis il se recourbe en crosse et se dirige vers l'arrière ; il comprend une première partie à paroi épaisse, puis une deuxième partie à paroi mince emplie 
d'œufs ; celle-ci se divise en deux utérus dirigés vers l'apex ; les extrémités des ovaires se trouvent au voisinage du bulbe œsophagien; les œufs, ovales et aplatis sur une face, sont embryonnés au moment de la ponte.

Le corps des femelles s'amincit brusquement légèrement en dessous de l'anus et se prolonge par une queue très longue et effilée.

Principales dimensions d'une femelle mûre longue de $2.560 \mu$.

Largeur maximum : $375 \mu$; cavité buccale: $10 \mu$ de profondeur; œsophage : $370 \mu$; anneau nerveux, pore excréteur et vulve situés respectivement à $175 \mu, 525 \mu$ et $920 \mu$ de l'extrémité antérieure ; queue : $1000 \mu$; ailes latérales débutant à $140 \mu$ de l'extrémité antérieure et se terminant à $960 \mu$ de l'extrémité postérieure ; œufs : $150 \mu / 65 \mu$.

Chez le Synodontis ocellifer 294 BA, nous trouvons des femelles jeunes beaucoup moins grosses et possédant seulement deux ou trois œufs.

\section{Mâles.}

Comme celui des femelles, le corps des mâles se rétrécit brusquement en dessous de l'anus pour se terminer en une queue très longue et effilée. Environ au quart antérieur de sa longueur, la queue porte ventralement une grosse papille double qui est très saillante en vue latérale; le cloaque se trouve à l'extrémité d'un gros mamelon saillant ; ce mamelon porte trois paires de petites papilles arrondies, concentrées autour de l'ouverture cloacale (voir fig. 3, G).

Le spicule est court et aciculaire.

Principales dimensions d'un mâle long d'environ $1.150 \mu$ (l'extrémité antérieure étant coupée, nous ne pouvons donner sa longueur exacte).

Largeur maximum : $200 \mu$; œsophage : un peu plus de $210 \mu$; pore excréteur situé à un peu plus de $245 \mu$ de l'extrémité antérieure; spicule : $60 \mu$; queue : $460 \mu$; papille caudale située à $340 \mu$ de l'extrémité postérieure; ailes latérales débutant à environ $60 \mu$ de l'extrémité antérieure et se terminant à $300 \mu$ de l'extrémité postérieure.

\section{Discussion.}

L'espèce rappelle beaucoup, en particulier par la disposition et la forme des papilles cloacales, les Thélastomes parasites d'Invertébrés; par contre, la structure céphalique est identique à celle des Cithariniella et voisine également de celle d'Ichthyouris ou de certains Thelandros (voir Thelandros meridionalis Chabaud et Brygoo, 1962); le genre le plus proche reste donc Cithariniella, mais l'aspect des papilles cloacales est différent et nous n'avons pas ici de dents pharyngiennes striées; nous pensons donc qu'il est nécessaire de créer un nouveau genre que nous nommons Synodontisia. 
Diagnose.

Synodontisia n. g.: Oxyuridae, Pharyngodoninae. Bouche avec six lèvres, quatre lèvres submédianes larges et deux lèvres latérales étroites; quatre grosses papilles submédianes et deux amphides pédonculées; petite cavité buccale limitée par les parois amincies de l'œsophage ; cloaque placé sur un gros mamelon saillant; trois paires de petites papilles arrondies concentrées autour du cloaque et une grosse papille caudale double sur la queue. Parasite de Poissons.

Espèce type unique: Synodontisia thelastomoides n. sp.

\section{Conclusions}

Les deux genres Cithariniella et Synodontisia possèdent un certain nombre de caractères en commun : d'une part, la structure apicale, avec six lèvres dont deux latérales plus étroites, quatre papilles submédianes ovalaires et deux amphides pédonculées ; d'autre part, la queue très longue et effilée portant chez le mâle une paire de petites papilles contiguës ou fusionnées en une papille double impaire; elles appartiennent donc à un même groupe qu'il s'agit de placer dans la classification des Oxyuroidea.

Il existe actuellement à notre connaissance seulement trois autres espèces d'Oxyures de Poissons : toutes trois américaines, appartenant à trois genres différents.

Parmi celles-ci, l'espèce Travnema travnema Pereira, 1938, est très différente des espèces décrites ci-dessus par son aspect général trapu avec queue courte et sa structure œsophagienne : œsophage en deux parties d'égales diñiensions et sans appareil valvulaire ; de plus, la queue du mâle ne possède pas sur l'extrémité caudale la paire de petites papilles contiguës si caractéristique de ces espèces.

L'espèce Laurotravassoxyuris travassosi Vigueras en est plus proche par l'extrémité caudale du mâle et les œufs munis de longs filaments ; cependant, l'extrémité apicale est très différente.

L'espèce Ichthyouris ro Inglis, 1962, possède une extrémité apicale à six lèvres et des amphides pédonculées rappelant celles de Cithariniella et Synodontisia, mais l'extrémité caudale du mâle présente de larges ailes et des pièces chitinoïdes tout à fait particulières et on ne retrouve pas la paire de papilles caudales de ces genres.

En conclusion, il n'existe donc pas de raison particulière de réunir toutes les espèces parasites de Poissons dans un même groupe et nous considérerons seulement les deux genres Cithariniella et Synodontisia.

Ils présentent, surtout Synodontisia, beaucoup de ressemblances avec certains Thelastomatidae d'Insectes : la cuticule à stries espacées et fortement marquées, produisant une annélation superficielle, l'extrémité postérieure du mâle très fine avec une paire de papilles caudales très proches ou fusionnées, situées sur une petite éminence. La disposition et la forme des papilles cloacales de Synodontisia thelastomoides rappellent beaucoup celles que l'on rencontre chez certains Thelastoma ou Cephalobellus (voir, par exemple, Thelastoma periplaneticola Basir, 1942 ou Cephalobellus potosiae Leibersperger, 1960 dans Leibersperger, 1960). Enfin, des œufs munis à leurs pôles de 
longs filaments formant des écheveaux qui emplissent les utérus se trouvent, par exemple, chez certains Binema.

Ceci nous a fait supposer un instant que nous avions affaire en réalité à des Oxyures d'Insectes ingérés par les Poissons, en même temps que leurs hôtes, mais les deux genres présentent une structure apicale qui n'a pas été rencontrée, à notre connaissance, chez les Thelastomatidae; ceux-ci ont, en effet, en règle générale, huit papilles au cycle externe; de plus, comme le signale Khalil, la localisation intestinale des parasites et leur abondance font écarter cette hypothèse. Enfin, certains Poissons parasités, comme Citharinus citharus et Distichodus brevipinnis, ont un régime alimentaire herbivore (voir Blache, 1964).

Nous considérons donc que ces espèces sont bien des Oxyures de Poissons. Leur aspect général, et principalement la forme de la queue du mâle, les place dans les Pharyngodoninae. En raison de leurs grandes ressemblances avec les Oxyures d'Insectes, nous supposons qu'elles représentent une lignée indépendante issue par capture des Thelastomatidae et ayant peu évolué depuis.

Nous remarquons que certains Pharyngodoninae de Reptiles carnivores, appartenant au genre Thelandros, par exemple, présentent des ressemblances avec nos espèces et ont également un aspect qui rappelle les Oxyures d'Insectes par leur striation cuticulaire annelée et la forme de la queue du mâle ; nous supposons donc qu'ils forment également un rameau dérivant directement de Thelastomatidae. On sait que la séparation entre Thelastomatidae et Pharyngodoninae, basée, en principe, sur le nombre de papilles céphaliques, n'est admise que par raison de commodité, certains Oxyures d'Invertébrés pouvant n'avoir que quatre papilles céphaliques; les Pharyngodoninae forment en réalité un ensemble de genres polyphylétiques, réunissant des rameaux issus, à plusieurs reprises, des Oxyures d'Insectes.

\section{Bibliographie}

Blache (J.), avec la collaboration de Miton (F.), Stauch (A.), Iltis (A.), Loubens (G.), 1964. - Les poissons du bassin du Tchad et du bassin adjacent du Mayo Kebbi. Etude systématique et biologique. O.R.S.T.O.M. Paris, 1964, 483 pp.

Chabaud (A.-G.) et Brygoo (E. R.), 1962. - Nématodes parasites de caméléons malgaches. Ann. Parasit., 37, 4, 569-602.

INGLIS (W. G.), 1962. - Ichthyouris ro gen. et sp. nov. (Nematoda): an Oxyurid from a Freshwater Fish. J. Helm., 36, 1/2, 45-50.

Khalil (L. F.), 1964. - Cithariniella citharini gen. et sp. nov. (Nematoda): an Oxyurid from a Freshwater Fish, Citharinus citharus in the Sudan. J. Helm., 38, 1/2, 41-46.

Leibersperger (E.), 1960. - Die Oxyuroidea der europäischen Arthropoden. Parasitologische Schriftenreihe, 11, 1-150.

Pereira (C.), 1938. - Travnema travnema n. g. e n. sp. (Nematoda: Oxyuridae), parasito de Curimatus elegans (Pisces: Characinidae) no nordeste brasileiro. Livro Jubilar Prof. L. Travassos, 381-384.

Perez Vigueras (I.), 1938. - Nota sobre algunos nematodes parasitos nuevos de Cuba. Livro Jubilar Prof. L. Travassos, 501-508. 